\title{
The Relationship between Urinary 8-Hydroxydeoxyguanosine and Metabolic Risk Factors in Asymptomatic Subjects
}

\author{
Michiaki Miyamoto a, b Kazuhiko Kotani ${ }^{a} \quad$ Shun Ishibashi $^{b} \quad$ Nobuyuki Taniguchi $^{a}$ \\ a Department of Clinical Laboratory Medicine and ${ }^{\mathrm{b}}$ Department of Medicine, Division of Endocrinology and \\ Metabolism, Jichi Medical University, Shimotsuke, Japan
}

\section{Key Words}

Urinary 8-hydroxydeoxyguanosine $\cdot$ Body mass index

Obesity $\cdot$ Triglyceride $\cdot$ High-density lipoprotein cholesterol

\begin{abstract}
Objective: The aim of this study was to investigate the relationship between metabolic risk factors and oxidative stress using urinary 8-hydroxydeoxyguanosine $(8-\mathrm{OHdG})$, a recently utilized biological marker, in asymptomatic subjects. Methods: Ninety subjects (males/females $=30 / 60$; mean age $=52$ years), who were nonsmoking, nondiabetic and not on any medicine, were enrolled in the study. The body mass index, blood pressure, total cholesterol, triglyceride (TG), high-density lipoprotein cholesterol (HDL-C) and glucose as well as urinary $8-\mathrm{OHdG}$ and creatinine were measured. $\boldsymbol{R e}$ sults: The median level of $8-\mathrm{OHdG}$ was $9.3 \mathrm{ng} / \mathrm{mg}$ creatinine (interquartile range: 5.8-23.2). TG (Pearson's correlation: $r=$ $0.262, p=0.013)$ and HDL-C $(r=-0.259, p=0.014)$ showed a significant correlation with 8-OHdG. A multiple linear regression analysis adjusted for all the variables revealed that only TG had an independently significant and positive correlation with 8 -OHdG $(\beta=0.231, p=0.046)$. Conclusion: The data in this population suggest that, among metabolic risk factors, hypertriglyceridemia may be weakly but significantly associated with hyperoxidative stress as assessed by $8-\mathrm{OHdG}$.
\end{abstract}

Copyright $\odot 2011$ S. Karger AG, Basel

\section{KARGER}

Fax +4161306 1234

E-Mail karger@karger.ch

www.karger.com
(C) 2011 S. Karger AG, Basel

1011-7571/11/0202-0187\$38.00/0

Accessible online at:

www.karger.com/mpp

\section{Introduction}

Obesity, hypertension and dyslipidemia are regarded as metabolic risk factors for atherosclerotic diseases; therefore, understanding the pathophysiology of metabolic risk factors is a key to controlling atherosclerosis [1]. The number of individuals with such metabolic risk factors is increasing [2]. Oxidative stress is generally considered to play a crucial role in these metabolic conditions [3]. Oxidative stress is a direct and/or indirect initiator of the oxidation of molecules such as lipids, proteins and nucleic acids as well as endothelial dysfunction $[3,4]$. This can be seen in arthrosclerosis-free individuals, and furthermore, subsequent atherosclerotic formation causes a vicious circle via oxidative stress induced at atherosclerotic sites [3, 4]. Therefore, it is important to evaluate oxidative stress in a primary risk assessment for the prevention of atherosclerotic diseases, and more studies using oxidative stress markers are needed in preventative settings.

There are presently several methods to measure oxidative stress. 8-hydroxydeoxyguanosine (8-OHdG) is generated from guanine in DNA bases by oxidative stress $[5,6]$. The measurement of $8-\mathrm{OHdG}$ excreted in urine quantitatively may reflect systemic oxidative stress $[5,6]$. While the respective features of the oxidative stress markers remain incompletely established, $8-\mathrm{OHdG}$ is known 
Table 1. Clinical characteristics of the subjects

\begin{tabular}{lcc}
\hline Variable & Values & $\begin{array}{l}\text { Range } \\
\text { min-max }\end{array}$ \\
\hline Age, years & $52 \pm 7$ & $40-69$ \\
Females/males & $60 / 30$ & \\
BMI, kg/m ${ }^{2}$ & $25.2 \pm 2.8$ & $19.8-29.9$ \\
Systolic blood pressure, mm Hg & $132 \pm 16$ & $106-168$ \\
Diastolic blood pressure, mm Hg & $78 \pm 8$ & $60-96$ \\
Glucose, mmol/l & $5.0 \pm 0.7$ & $3.9-6.0$ \\
Total cholesterol, mmol/l & $5.4 \pm 0.7$ & $3.7-7.7$ \\
HDL-C, mmol/l & $1.5 \pm 0.4$ & $0.9-2.5$ \\
Triglyceride, mmol/l & $1.1(0.9-1.8)$ & $0.5-3.4$ \\
Urinary 8-OHdG, ng/mg creatinine & $9.3(5.8-23.2)$ & $0.90-48.0$ \\
& &
\end{tabular}

Data are the mean \pm standard deviation, median (interquartile range) or subject number.

to have a unique ability to evaluate a DNA injury which participates in the pathophysiology of metabolic risk factors and atherosclerotic diseases [7, 8]. In addition to this feature, as the improvement of analytical methods has made measurements easy and reliable [9], 8-OHdG has recently become one of the most widely used markers of oxidative stress $[7,8]$. Despite this, there are few studies that examine simultaneously and comparatively the relationship of 8-OHdG with some metabolic risk factors, while the association between urinary $8-\mathrm{OHdG}$ and single diseases such as hypertension [10] or diabetes mellitus [11-13] has been reported. The aim of this study was to investigate the relationship between metabolic risk factors and oxidative stress using urinary $8-\mathrm{OHdG}$, a recently utilized biomarker, in asymptomatic subjects.

\section{Subjects and Methods}

A total of 90 asymptomatic subjects (females: 60, males: 30; mean age: $52.2 \pm 7.6$ years, range: $40-69$ ) participated in this study. Consenting participants were recruited from among community-dwelling volunteers in a health education class setting. The ethics committee at Jichi Medical University approved the study. All eligible participants were nonsmoking and nondiabetic individuals who had no known history of cardiovascular, cerebrovascular, kidney or liver disease. In addition, they were not currently taking any medications, including antioxidant agents. Concomitant with self-reports, the absence of diabetes was initially checked by plasma glucose concentrations in a fasting blood test $(<6.1 \mathrm{mmol} / \mathrm{l}$, the level as previously reported [14]).

A fasting blood sample and spot urinary sample were collected from each individual in the morning before breakfast (7:00-9:00 a.m.). The body mass index (BMI) was calculated as the weight divided by the square of height $\left(\mathrm{kg} / \mathrm{m}^{2}\right)$. Systolic and diastolic blood pressure were measured 3 times in the arm of each patient in the seated position after a rest for a minimum of $5 \mathrm{~min}$, and the average values of these 3 measurements were used in the analyses. Serum total cholesterol and triglyceride (TG), plasma glucose and urinary creatinine were measured by enzymatic methods supplied by Eiken Kagaku Co. Ltd. (Tokyo, Japan) and Shino-Test Co. Ltd. (Tokyo, Japan), respectively. High-density lipoprotein cholesterol (HDL-C) was measured by a direct assay (Kyowa Medics Co. Ltd., Tokyo, Japan). Urinary samples were centrifuged at $300 \mathrm{~g}$ for $10 \mathrm{~min}$ and the supernatant after dilution was applied to an enzyme-linked immunosorbent assay kit with a highly sensitive monoclonal antibody to 8-OHdG (N45.1 [15]) supplied by the Japan Institute for Aging (Shizuoka, Japan). The creatinine value was used for correction of urinary $8-\mathrm{OHdG}$ concentrations. A stable correlation between spot urine levels and 24-hour excretion of 8-OHdG had already been established [16]. The inter- and intra-assay coefficients of variation were 2.1 and $4.5 \%$, respectively.

Data are expressed as the mean \pm standard deviation or median and interquartile range. Pearson's correlation test and a multiple linear regression analysis adjusted for all the measured variables were used to observe the correlations between 8-OHdG and the other variables. In these analyses, the log-transformed values of 8-OHdG and TG were used because of their skewed distributions. $\mathrm{p}<0.05$ was considered to be significant. The statistical analyses were performed with the Statistical Package for Social Science (SPSS) version 11.0 for Windows (SPSS Inc., Chicago, Ill., USA).

\section{Results}

The subjects' clinical characteristics are presented in table 1. Simple correlations demonstrated that the levels of TG (positively) and HDL-C (inversely) were significantly correlated to those of $8-\mathrm{OHdG}$, respectively, as shown in table 2. Similar results were detected when TG and 8-OHdG were not log-transformed: there was a significant and positive correlation between TG and 8 -OHdG (Spearman rank correlation test; $\mathrm{r}=0.245, \mathrm{p}=$ $0.020)$ as well as a significant and inverse correlation between HDL-C and 8-OHdG ( $\mathrm{r}=-0.324, \mathrm{p}=0.001)$. A multiple linear regression analysis, adjusted for age, sex and all the measured variables, revealed only TG to independently show a significant and positive correlation with 8-OHdG. No other variables showed any significant correlation.

\section{Discussion}

A mong several metabolic risk factors, TG was the only metabolic risk factor which weakly but significantly and independently correlated with $8-\mathrm{OHdG}$ in the asymp- 
Table 2. Correlations between urinary $8-\mathrm{OHdG}$ and all the measured variables

\begin{tabular}{lrlrl}
\hline Variable & $\mathrm{r}$ & $\mathrm{p}$ & \multicolumn{1}{c}{$\beta$} & $\mathrm{p}$ \\
\hline Age, years & 0.176 & 0.097 & 0.174 & 0.096 \\
Male gender, n & -0.032 & 0.766 & -0.018 & 0.868 \\
BMI, kg/m² & 0.147 & 0.167 & 0.133 & 0.233 \\
Systolic blood pressure, & & & & \\
$\quad$ mm Hg & 0.141 & 0.185 & -0.070 & 0.610 \\
Diastolic blood pressure, & & & & \\
$\quad$ mm Hg & 0.147 & 0.166 & 0.133 & 0.292 \\
Glucose, mmol/l & -0.107 & 0.318 & -0.123 & 0.249 \\
Total cholesterol, mmol/l & -0.155 & 0.146 & -0.136 & 0.201 \\
HDL-C, mmol/l & -0.259 & $0.014^{*}$ & -0.153 & 0.185 \\
Triglyceride, mmol/l & 0.262 & $0.013^{*}$ & 0.231 & $0.046^{*}$ \\
\hline
\end{tabular}

8-OHdG and triglyceride were log-transformed.

${ }^{*} \mathrm{p}<0.05 . \mathrm{r}=$ Pearson's correlation coefficient; $\beta=$ multiple linear regression coefficient.

tomatic subjects. The results of the present study are consistent with previous findings that show the correlation between TG and hyperoxidative stress using different markers: plasma 8-epi-prostaglandin F2- $\alpha$ [17]; oxygen radical generation and superoxide scavenging activity by monocytes [18]; reactive oxygen species-release by leukocytes, lipid hydroperoxides and plasma antioxidant enzymes [19]. Our present data appear to be meaningful in reinforcing and expanding these observations by establishing the use of a recent biological marker.

The significant correlation between increased levels in TG and such oxidative stress markers is also valuable because TG has not necessarily been a representative metabolic risk factor for atherosclerosis in comparison to other atherosclerotic factors such as blood pressure and cholesterol $[20,21]$. The data from these studies, including the present findings, may contribute to a better understanding of the underlying role of TG in the prevention of atherosclerosis. While the detailed molecular pathways involved are yet to be determined, TG has been suggested to trigger a hyperoxidative stress environment [1719]. These reports imply that TG may stimulate the production of reactive oxygen species and impair the antioxidant defense system $[18,19]$. Moreover, some reports describe an influence of TG even greater than that of glucose [18] and cholesterol [19]. An additional mechanistic view of the relationship between TG and oxidative stress suggests that the effects of obesity on TG for oxidative stress may occur because an excessive accumulation of TG in adipocytes accompanying hypertriglyceridemia is found in obesity [22]. However, BMI was not significantly correlated with $8-\mathrm{OHdG}$ in the present study. This mechanism, even though true, might be partly weakened by the fact that the Japanese (especially communitydwelling individuals such as the present study participants) have a lower BMI than Caucasian populations [23].

Although the correlations between the other oxidative stress markers and metabolic risk factors, except for TG, have previously been documented $[10,11]$, the present study showed weak correlations between 8-OHdG and BMI, systolic blood pressure, diastolic blood pressure, glucose, total cholesterol and HDL-C. This may be partly explained by the minor abnormality in the degree of blood pressure, plasma glucose and cholesterol in our study population in a preventative setting. Furthermore, this could affect the significant but weak correlation of TG to 8-OHdG. Although a reciprocal association between TG and HDL-C is often observed in individuals with insulin resistance and metabolic syndrome, the lack of a significant correlation of HDL-C to 8-OHdG might also be partially explained by this population. In addition, the response to the various metabolic risk factors can differ according to oxidative stress markers. Dietary habits [24] and mental states [25] may be related to the state of oxidative stress. Residual chronic diseases unidentified in the present study may also affect the measurement. These points are all considered to be limitations of this study, and therefore comparative studies using various markers, including more detailed assessments on dietary, mental and physical aspects, should be performed in future research.

\section{Conclusion}

TG was the metabolic risk factor found to independently have a significant and positive correlation to urinary 8 -OHdG, a recently utilized biological marker of oxidative stress, in an asymptomatic population. Future studies must determine whether the management of TG can effectively prevent the production of oxidative stress.

\section{Acknowledgements}

This study was supported in part by a Grant-in-Aid for Scientific Research from the Ministry of Education, Culture, Sports, Science and Technology of Japan (K.K.) and the Foundation for the Development of the Community, Japan. 


\section{References}

$>1$ Alberti KG, Zimmet P, Shaw J: IDF Epidemiology task force consensus group. The metabolic syndrome - a new worldwide definition. Lancet 2005;366:1059-1062.

$>2$ Aronne LJ, Isoldi KK: Overweight and obesity: key components of cardiometabolic risk. Clin Cornerstone 2007;8:29-37.

$\checkmark 3$ Furukawa S, Fujita T, Shimabukuro M, Iwaki M, Yamada Y, Nakajima Y, Nakayama O, Makishima M, Matsuda M, Shimomura I: Increased oxidative stress in obesity and its impact on metabolic syndrome. J Clin Invest 2004;114:1752-1761.

4 Giannini C, de Giorgis T, Scarinci A, Cataldo I, Marcovecchio ML, Chiarelli F, Mohn A: Increased carotid intima-media thickness in pre-pubertal children with constitutional leanness and severe obesity: the speculative role of insulin sensitivity, oxidant status, and chronic inflammation. Eur J Endocrinol 2009;161:73-80.

$\checkmark 5$ Kasai H, Hayami H, Yamaizumi Z, Saito H, Nishimura S: Detection and identification of mutagens and carcinogens as their adducts with guanosine derivatives. Nucleic Acids Res 1984; 12:2127-2136.

$\checkmark 6$ Loft S, Poulsen HE: Cancer risk and oxidative DNA damage in man. J Mol Med 1996; 74:297-312.

7 Piconi L, Quagliaro L, Ceriello A: Oxidative stress in diabetes. Clin Chem Lab Med 2003; 41:1144-1149.

-8 Valavanidis A, Vlachogianni T, Fiotakis K, Valavanidis A, Vlachogianni T, Fiotakis C: 8-hydroxy-2'-deoxyguanosine (8-OHdG): a critical biomarker of oxidative stress and carcinogenesis. J Environ Sci Health C Environ Carcinog Ecotoxicol Rev 2009;27:120139.

-9 Kasai H, Kawai K, Li Y: Analysis of 8-OH-dG and $8-\mathrm{OH}-\mathrm{Gua}$ as biomarkers of oxidative stress. Genes Environ 2008;30:33-40.

10 Negishi H, Ikeda K, Kuga S, Noguchi T, Kanda T, Njelekela M, Liu L, Miki T, Nara Y, Sato T, Mashalla Y, Mtabaji J, Yamori Y: The relation of oxidative DNA damage to hypertension and other cardiovascular risk factors in Tanzania. J Hypertens 2001;19:529-533.
11 Nishikawa T, Sasahara T, Kiritoshi S, Sonoda K, Senokuchi T, Matsuo T, Kukidome D, Wake N, Matsumura T, Miyamura N, Sakakida M, Kishikawa H, Araki E: Evaluation of urinary 8-hydroxydeoxy-guanosine as a novel biomarker of macrovascular complications in type 2 diabetes. Diabetes Care 2003; 26:1507-1512.

12 Sena CM, Nunes E, Louro T, Proença T, Seiça RM: Endothelial dysfunction in type 2 diabetes: effect of antioxidants. Rev Port Cardiol 2007;26:609-619.

13 Dong QY, Cui Y, Chen L, Song J, Sun L: Urinary 8-hydroxydeoxyguanosine levels in diabetic retinopathy patients. Eur J Ophthalmol 2008; 18:94-98.

14 The Expert Committee on the Diagnosis and Classification of Diabetes Mellitus: Report of the Expert Committee on the Diagnosis and Classification of Diabetes Mellitus. Diabetes Care 1997;20:1183-1197.

15 Toyokuni S, Tanaka T, Hattori Y, Nishiyama Y, Yoshida A, Uchida K, Hiai H, Ochi H, Osawa T: Quantitative immunohistochemical determination of 8-hydroxy-2'-deoxyguanosine by a monoclonal antibody N45.1: its application to ferric nitrilotriacetate-induced renal carcinogenesis model. Lab Invest 1997;76:365-374.

16 Poulsen HE, Loft S, Prieme H, Vistisen K, Lykkesfeldt J, Nyyssonen K, Salonen JT: Oxidative DNA damage in vivo: relationship to age, plasma antioxidants, drug metabolism, glutathione-S-transferase activity and urinary creatinine excretion. Free Radic Res 1998;29:565-571.

17 Katsuki A, Sumida Y, Urakawa H, Gabazza EC, Murashima S, Nakatani K, Yano Y, Adachi Y: Increased oxidative stress is associated with serum levels of triglyceride, insulin resistance, and hyperinsulinemia in Japanese metabolically obese, normal-weight men. Diabetes Care 2004;27:631-632.

18 Pronai L, Hiramatsu K, Saigusa Y, Nakazawa $\mathrm{H}$ : Low superoxide scavenging activity associated with enhanced superoxide generation by monocytes from male hypertriglyceridemia with and without diabetes. Atherosclerosis 1991;90:39-47.
19 Araujo FB, Barbosa DS, Hsin CY, Maranhão RC, Abdalla DS: Evaluation of oxidative stress in patients with hyperlipidemia. Atherosclerosis 1995; 117:61-71.

20 Hodis HN, Mack WJ, Azen SP, Alaupovic P, Pogoda JM, LaBree L, Hemphill LC, Kramsch DM, Blankenhorn DH: Triglyceride- and cholesterol-rich lipoproteins have a differential effect on mild/moderate and severe lesion progression as assessed by quantitative coronary angiography in a controlled trial of lovastatin. Circulation 1994;90:42-49.

21 Iso H, Naito Y, Sato S, Okamura T, Sankai T, Shimamoto T, Iida M, Komachi Y: Serum triglycerides and risk of coronary heart disease among Japanese men and women. Am J Epidemiol 2001;153:490-499.

22 Keaney JF Jr, Larson MG, Vasan RS, Wilson PW, Lipinska I, Corey D, Massaro JM, Sutherland P, Vita JA, Benjamin EJ: Obesity and systemic oxidative stress: clinical correlates of oxidative stress in the Framingham study. Arterioscler Thromb Vasc Biol 2003; 23:434-439.

23 McNeely MJ, Boyko EJ, Shofer JB, NewellMorris L, Leonetti DL, Fujimoto WY: Standard definitions of overweight and central adiposity for determining diabetes risk in Japanese Americans. Am J Clin Nutr 2001; 74:101-107.

24 Thompson HJ, Heimendinger J, Haegele A, Sedlacek SM, Gillette C, O’Neill C, Wolfe P, Conry C: Effect of increased vegetable and fruit consumption on markers of oxidative cellular damage. Carcinogenesis 1999;20: 2261-2266.

25 Cernak I, Savic V, Kotur J, Prokic V, Kuljic B, Grbovic D, Veljovic M: Alterations in magnesium and oxidative status during chronic emotional stress. Magnes Res 2000;13:2936. 